\title{
Relación de la Asimetría Bilateral y el Déficit Bilateral con la Velocidad del Cambio de Dirección en Atletas Cadetes de Karate: Un estudio Piloto Relationship of Bilateral Asymmetry and Bilateral Deficit with the Change of Direction Speed in Cadet Karate Athletes: A Pilot Study \\ *,**Alex Ojeda-Aravena; *,**jairo Azócar-Gallardo; ***,****Tomás HerreraValenzuela, **José Manuel García-García \\ *Universidad de Los Lagos (Chile); **U niversidad de Castilla La M ancha (España); ***Universidad SantoTomás (Chile); $* * * *$ Universidad de Santiago (Chile)
}

Resumen. Antecedentes: La Asimetría Bilateral ( $A B$ ) y el Déficit Bilateral (DBL) y su potencial relación negativa con el rendimiento físico es actual mente estudiada, aunque la relación con la velocidad del cambio de dirección (CO DS) y el sprint lineal (5-M) en atletas de karate se desconoce. Objetivo: Examinar las correlaciones entre laAB y el DBL con el CO DS y 5-M, así como entre el salto horizontal con el CODS y 5-M. Métodos: Diez atletas hombres de categoría cadetes participaron voluntariamente de este estudio. Entre las habilidades físicas eval uadas se incluyeron: salto horizontal bilateral (SBJ), salto horizontal unilateral derecho (RSBJ), salto horizontal unilateral izquierdo (LSBJ), CODS y 5-M. Posteriormente, laAB y el DBL fueron calculados. Resultados: Se reportó una bajaAB $(5.17 \pm 3.61 \%)$ y presenciadeD BL $(-36.9 \pm 6.2 \%)$. Correlaciones moderadas entre laAB con CODS $\left(r=-.38 ; R^{2}=.15 \% ; p=.30\right)$ y $5-M\left(r=-.47 ; R^{2}=.22 \% ; p=.19\right)$ y entre $D B L$ con $5-$ $M\left(r=.51 ; R^{2}=.26 \% ; p=.16\right)$ y bajas con CODS $\left(r=.20 ; R^{2}=.04 \% ; p=.60\right)$. Correlaciones significativas $(p<.05)$ entre CODScon RSBJ $\left(r=-.92 ; R^{2}=.85 \% ; p=.00\right)$, SB) $\left(r=-.87 ; R^{2}=.75 \% ; p=.00\right)$ y LSB $\left(r=-.82 ; R^{2}=.67 \% ; p=.00\right)$. Conclusiones: $L a A B$ y el DBL se relacionarían negativamente con el CODSy $5-M$. Aunque, el CODS se relaciona con la fuerza explosiva horizontal bilateral y unilateral.

Palabras claves: Artes marciales, Hombres, Atletas, Fuerza M uscular.

Abstract. Background: Bilateral asymmetry (AB) and Bilateral Deficit (DBL) and their potential negative relationship with physical performance is currently studied, although the relationship with change of direction speed (CODS) and linear sprint (5-M) in karate athletes is unknown. Aim: To examine correlations between AB and DBL with CODS and 5-M, as well as between horizontal jump with CODS and 5-M. M ethods: Ten male cadet athletes voluntarily participated in this study. The physical abilities assessed included: bilateral horizontal jump (SBJ), right unilateral horizontal jump (RSBJ), left unilateral horizontal jump (LSBJ), CODS and 5-M. Subsequently, AB and DBL were calculated. Results: Low $A B(5.17 \pm 3.61 \%)$ and presence of DBL $(-36.9 \pm 6.2 \%)$ were reported. M oderate correlations between $A B$ with $C O D S\left(r=-.38 ; R^{2}=.15 \% ; p=\right.$ .30) and $5-M\left(r=-.47 ; R^{2}=.22 \% ; p=.19\right)$ and between DBL with $5-M\left(r=.51 ; R^{2}=.26 \% ; p=.16\right)$ and low with CODS $\left(r=.20 ; R^{2}=.04 \% ; p=.60\right)$. Significant correlations $(p<.05)$ between CO DS with RSB $\left(r=-.92 ; R^{2}=.85 \% ; p=.00\right)$, SBJ $\left(r=-.87 ; R^{2}=.75 \% ; p=.00\right)$ and LSBJ $\left(r=-.82 ; R^{2}=.67 \% ; p=.00\right)$. Conclusions: $A B$ and $D B L$ would be negatively related to CODS and 5-M. However, CODS is related to bilateral and unilateral horizontal explosive strength.

Keywords: Martial Arts, Male, Athletes, Muscular Strength.

\section{Introducción}

El fenómeno de la Asimetría Bilateral (AB) o asimetría inter-extremidades referido a las diferencias en el rendimiento o función de un miembro con respecto al otro (Bishop et al., 2019b; Bishop et al. , 2019c), esun tema de estudio de interés en los últimos años. La literatura establece diferentes clasificaciones para estas diferencias bilaterales que incluyen: dominante vs. nodominante, fuerte vs. débil, izquierda vs. derecha, le-

Fecha recepción: 09-12-20. Fecha de aceptación: 09-03-21

Alex Ojeda-Aravena

alex.ojeda@ulagos.cl sionado vs. no-lesionado (Bishop et al., 2017). Para efectos del rendimiento deportivo, es importante distinguir entre lateralidad (dominio de «habilidad») y dominio de la fuerza (es decir, la extremidad que demuestra cualidades de fuerza superiores en una tarea determinada) (Meyers et al., 2017). En este sentido, dada la innegable influencia de la fuerza y la potencia muscular en el rendimiento físico de los atletas, la $A B$ ha sido evaluada utilizando diversas tareas de fuerza que incluyen: sentadilla profunda, dinamometría isocinética y tareas isométricas como sentadilla isométrica (Bishop et al. , 2018a; Bishop et al., 2019c) y de manera indirecta através de saltos verticales (Sannicandro et al. , 2012), horizontales (del inglés: Standing Broad Jump o SBJ) y 
laterales (Lockie et al., 2014; Soñén et al., 2021). Además, los tópicos de estudio se han centrado principalmente en establecer la relación entre la AB y incremento del riesgo de lesión (> $15 \%$ ) (Konset al., 2021) y de manera creciente durante los últimos años sobre su potencial relación negativa con el rendimiento físico 0 deportivo (Bishop et al., 2018a; Bishop et al., 2018b; Bishop etal., 2019b; Konset al., 2020; Konset al., 2021).

De acuerdo con lo anterior, los estudios que analizan esta problemática han predominado mayoritariamente en los deportes colectivos (Madruga-Pareraet al. , 2020; Bishop et al., 2019b; Bishop, et al., 2019d; Lockieet al., 2014; Hoffman et al., 2007) y de manera reciente en los deportes de combate (Kons et al., 2020; Kons et al., 2021; Soñén et al., 2021) con resultados controversiales. En tal sentido, por ej. los autores Bishop et al. (2019d) reportaron correlacionesinversassignificativas $(p<.05)$ entre laAB evaluada mediante $D$ rop Jump con un menor rendimiento en sprint de $10 \mathrm{~m}$ a $30 \mathrm{~m}$ y lavelocidad del cambio de dirección (del inglés: Change of Direction Speed o CODS) en mujeres adultasfutbolistas. También, Bishop et al. (2019b) documentar on correlaciones inversas significativas $(p<.05)$ entre la AB mediante CounterMovement Jump (CMJ) con una disminución del rendimiento en $10 \mathrm{~m}$ sprint y CODS (a través de la prueba 505) en futbolistas de élite de diferentes categorías de edad. En jugadores de balonmano, los autores M adruga Pareraet al. (2020) documentaron correlacionesinversas significativas $(p<.05)$ entre la $A B$ evaluada mediante SB], salto lateral, CMJ y en tareas específicas de CODS con un menor rendimiento en CO DSy en sprint en 20 m. Por otro lado, Hoffman et al. (2007), si bien observó una $A B$ en $C M J$, los autores no reportaron disminuciones significas $(p>.05)$ en el rendimiento en CODS. Del mismo modo, los autores Lockie et al. (2014) no documentaron correlaciones significativas entre la $A B$ de salto vertical, horizontal y lateral con el rendimiento del sprint 0 a $20 \mathrm{~m}$ y el CODS mediante las prueba T y 505 en hombres de diferentes deportes de equipo.

Específicamente, en deportes de combate en judo concretamente, Kons et al. (2020) documentaron durante una sesión de ejercicio físico, $A B$ entre las extremidades inferiores a través de ultrasonografía y $C M J$, además de correlaciones inversas significativas $(p<.05)$ con indicadores específicos de la prueba específica de alta-intensidad Special Judo Fitness Test. En esta misma línea, recientemente Kons et al. (2021) estudiaron la $A B$ utilizando CMJ, SBJ, y la fuerza de agarre en cuatro combates simulados de cuatro min. Entre los resultados documentaron incrementos significativos $(p<.05)$ en
laAB documantada partir del CMJ al finalizar el segundo combate $(p<.00)$. En karate, en atletas internacionales la presencia de $A B$ ha sido reportada mediante dinamometría isocinética, aunque sin diferencias significativas ( $p>.05)$ entre las extremidades inferiores (Scattone-Silva et al., 2012). También, recientemente Soñen et al. (2020) reportaron AB mediante el salto horizontal $(5.3 \pm 3.9 \%)$ y CMJ $(12.2 \pm 8.5 \%)$ en atletas de karate de nivel nacional e internacional.

Ahora bien, se ha sugerido que el CO DS es importante en los deportes de combate dado que los atletas de karate cambian continuamente de dirección, acelerando de manera lineal y en diferentes direcciones durante los combates (Herrera-Valenzuela et al., 2020; 0 jedaA ravena et al., 2020; Chaabeneet al., 2012). De hecho, los atletas de karate de élite y de mayor experiencia competitiva presentan un rendimiento superior en CODS que atletas de nivel nacional y de menor experiencia (de Quel et al., 2020). Además, el CO DS ha sido descrita como una habilidad física predictora del éxito competitivo en atletas de élite mujeres de karate (de Q uel et al., 2020) y estar relacionada con la fuerza explosiva, específicamente con las pruebas de salto SJ y CMJ (Herrera-Valenzuela et al., 2020). También, considerando el predominio de las técnicas ofensivas de mano $(84.4 \%)$, particularmente utilizando la mano adelantada o kizami tsuki (Chaabene et al., 2015; Tabben et al., 2018) seguido de las técnicas de patadas circulares o mawashi geri documentadas en combate (Chaabene et al., 2015; Tabben et al., 2018), sugieren una predominancia unilateral en la ejecución de las técnicas utilizadas, por lo que este predominio podría verse reflejado en una mayor $A B$ influyendo negativamente en el CODS.

Por otra parte, otro indicador utilizado a partir de la evaluación unilateral y de las evaluaciones bilaterales de la fuerza explosiva, es el Déficit Bilateral (DBL) (Sarabon et al., 2020; Bishop et al. 2019a). Término referido a que la diferencia entre la máxima fuerza producida con ambas extremidades (por ej. durante una contracción bilateral máxima) tiende a ser menor que la suma de la fuerza total producida por las extremidades izquierda y derecha de manera separada (Bishop et al., 2019a). Al respecto, la evidencia muestra correlaciones inversas significativas $(p<.05)$ entre una ma yor DBL mediante CMJ con un mayor rendimiento en CO DS (Bishop et al., 2019a), aunque su relación en atletas de karate se desconoce.

De acuerdo con lo anterior, este estudio puede ayudar a los entrenadores a comprender la influencia de la 
$A B$ y el DBL como herramientas evaluaciones prácticas relacionadas con el monitoreo, lamejoradel rendimiento y la prevención de lesiones utilizando métodos sencillos de evaluación a través del salto largo horizontal bilateral y unilateral basados en el supuesto de que la asimetría exacerbada entre las extremidades puede aumentar el riesgo de sufrir lesiones musculares (Kons et al. , 2021) y/ 0 afectar negativamente el rendimiento físico. En consecuencia, el propósito principal fue exa minar la correlación entre la Asimetría Bilateral y el Déficit Bilateral con la velocidad del cambio de dirección y el sprint en 0 a $5 \mathrm{~m}$, así como entre el salto horizontal con el velocidad del cambio de dirección y el sprint en 0 a $5 \mathrm{~m}$. Hipotetizamos que laAsimetría Bila teral y el Déficit Bilateral se correlacionarían negativa mente con el rendimiento de velocidad del cambio de dirección y el sprint en 0 a $5 \mathrm{~m}$ y que el salto horizontal bilateral y unilateral correlacionaría positivamente con el rendimiento en CODS y el sprint en 0 a $5 \mathrm{~m}$.

\section{Material y M étodo}

\section{Acercamiento experimental al problema}

Se utilizó un diseño correlacional transversal con selección de la muestra de manera no probabilística por conveniencia donde los atletas ejecutaron saltos bilaterales horizontales (SB) ) y unilaterales con la pierna izquierda (LSBJ) y derecha (RSBJ), las pruebas de velocidad del cambio de dirección mediante la prueba ZigZag (CODS) y sprint en 0 a $5 \mathrm{~m}(5-\mathrm{M})$. Durante la semana previa, una sesión de familiarización de las pruebas fue realizada a los atletas entregando las instrucciones y el tiempo necesario para practicar cada prueba tantas veces como fuese necesario, durante una hora para asegurar la familiarización de los procedimientos y reducir el efecto de aprendizaje. La sesión fue monitoreada por un profesional cualificado y discutido verbal mente con cada atleta para garantizar que ambas partes estuvieran satisfechas con los requisitos antes de la recopilación de datos. El reclutamiento de los atletas del estudio fue realizado el mes de abril del 2019 como parte de las evaluaciones previas del programa de especialización deportiva del Instituto Nacional Deportes de Chile (IND). Las sesiones de las evaluaciones fueron realizadas durante dos días consecutivos desde las 12:00 h a 16:00 h. El primer día se evaluaron las características antropométricas y posterior a 24 h, las variables del rendimiento físico (RF) de acuerdo con la intensidad muscular de cada prueba en el siguiente orden: SBJ, LSBJ, RSBJ, 5-M y CODS. Dos ensayos para cada prueba fueron realizados y el mejor puntaje obtenido fue utilizado para el análisis estadístico posterior. Posteriormente, a partir de la mejor distancia al canza da de las evaluaciones SBJ, LSB] y RSB] se calcularon los índices de la $A B$ y el $D B L$.

\section{Participantes}

Diez atletas hombres de karate (edad $14 \pm 1$ años; estatura $164 \pm 11 \mathrm{~cm}$; masa corporal $56 \pm 12 \mathrm{~kg}$; experiencia de entrenamiento $5 \pm 1$ años) de la categoría cadetes pertenecientes a una escuela de karate de nivel competitiva nacional e internacional de Puerto M ontt, adherida a la Federación Deportiva Nacional de karate de Chile y participantes al programa de especialización deportiva del Instituto Nacional del Deporte (IND) participaron voluntariamente del estudio. Para su inclusión, debieron cumplir con los siguientes criterios: (i) entrenamiento sistemático por más de 4 años, durante al menos tres veces por semanas, (ii) entrenamiento ininterrumpido previo a su inclusión en el estudio durante e» 6 meses, (iii) con ausencia de lesiones músculo-esqueléticas, (iv) encontrarse preparando competiciones o torneos organizados por la Federación Deportiva Nacional de karate de Chile, una organización reconocida por la World Karate Federation (WKF). Todos los atletas y/ o los familiares de los atletas menores de 18 años fueron informados previamente de los objetivos del estudio, los beneficios asociados, los procedimientos experimentales y los posibles riesgos mediante un consentimiento informado 0 asentamiento informado antes de las evaluaciones. El estudio se condujo de acuerdo con la declaración de Helsinki (World Medical Association, 2013).

\section{Instrumentos}

Evaluaciones antropométricas. La estatura $(\mathrm{cm})$ fue evaluada a través de un estadiómetro (Bodymeter 206) con precisión de un $\mathrm{mm}$ siguiendo los protocolos estándares (Caballero \& Díaz, 2003). Brevemente los atletas se ubicaron sin zapatos, con los talones unidos, espalda y glúteos tocando la superficie vertical del estadiómetro y la cabeza colocada en el plano de Frankfort. Lamasacorporal (MC) fueevaluadamediante escala eléctrica de impedancia bioléctrica (InBody120, tetrapolar 8-point tactile electrodes system, model BPM 040S12F07, Biospace, Inc. , USA, to $0.1 \mathrm{~kg}$ ) utilizando losprotocolos demediciones estándares(M cLester et al., 2018).

Velocidad de cambio de dirección (CO DS). Laprueba Zig-Zag fue utilizada para evaluar el rendimiento de 
CODS siguiendo las recomendaciones de Loturco et al. (2018). La prueba consistió en cuatro secciones de $5 \mathrm{~m}$ (20 m de velocidad lineal total) marcadas con conos en ángulos de 100으, que requieron que los atletas desaceleren y aceleren lo más rápido posible al rededor de cada cono. Comenzando desde una posición de pie con el pie delantero colocado $0.3 \mathrm{~m}$ detrás del primer par de fotocélulas (Brower Timing System, Salt Lake City, UT) con precisión de $0.001 \mathrm{~s}$. Se instruyó a los atletas completar la prueba lo más rápido posible, hasta cruzar el segundo par de puertas de tiempo, ubicado a $20 \mathrm{~m}$ de la línea de salida (Little $\&$ W illiams, 2005). El tiempo más rápido de los dos ensayos fue utilizado para el análisis estadístico posterior.

Fuerza explosiva horizontal bilateral. El salto horizontal bilateral (SB)) fue utilizado como medida indirecta de la fuerza explosiva horizontal siguiendo las recomendaciones previas de Loturco et al., (2018). Brevemente se solicitó a los atletas que se pararan con los dedos de los pies alineados con un marcador negro colocado en el suelo. Los atletas recibieron instrucciones de realizar un salto horizontal después de ponerse en cuclillas a una profundidad autoseleccionada, con el objetivo de saltar lo más adelante posible. Se permitió un balanceo del brazo para esta prueba debido a la mayor dificultad. Se real izaron dos ensayos con un descanso de dos min entre intentos y el mejor intento fue incluido en el análisis estadístico posterior.

Fuerza explosiva horizontal unilateral. Los saltos horizontal unilateral izquierdo (LSBJ) y Derecho (RSBJ) fueron utilizados como medidas indirectas de la fuerza explosiva horizontal de las extremidades inferiores izquierda y derecha respectivamente, utilizando las recomendaciones de Lockie et al. (2014). Se solicitó a los atletas colocar los dedos de ambos pies en la parte posterior de la línea de inicio, antes de equilibrar la extremidad que se evaluó. Con un movimiento simultáneo del brazo y agachado, el atleta saltó lo más adelante posible, despegando de una pierna, antes de aterrizar en dos pies. Los atletas tuvieron que «pegar» el aterrizaje para que se contara el ensayo. Si el atleta no hizo esto, se desestimó el ensayo y se intentó otro. Se instruyó a los atletas para que usaran un contra movimiento, y no se impusieron restricciones en los ángulos corporales al canzados durante la fase preparatoria del sal to 0 el movimiento del brazo utilizado. La distancia se midió al $0.01 \mathrm{~m}$ más cercano usando una cintamétricaestándar, perpendicular desde el frente de la línea de inicio hasta la parte posterior del talón trasero en el aterrizaje. Se realizaron dos ensayos para cada prueba y el mejor en- sayo se utilizó para el análisis estadístico posterior.

Asimetría Bilateral (AB). LaAsimetría Bilateral entre las extremidades inferiores (magnitud) fue cuantificado como el porcentaje de diferencia (\%AB) entre el miembro más fuerte y el más débil (\%AB) utilizando la ecua ción 1 propuesta por Impellizzeri et al. (2007) y verificada por Bishop et al. (2018b). Se calculó el índice de asimetría de la extremidad más fuerte y más débil para el rendimiento de la distancia al canzada en SBJ.

\section{Ecuación 1}

$$
\% \mathrm{AB}=\frac{\text { (extremidad fuerte }- \text { extremidad débil) }}{\text { extremidad fuerte }} * 100
$$

Déficit Bilateral (DBL). El Déficit Bilateral fue cuantificado mediante el índice Bilateral (IB) que es la rela ción entre los valores bilaterales y unilaterales del salto horizontal bilateral, a partir de los valores unilateral izquierdo, unilateral derecho y bilateral de las pruebas SB], como puede verse en la Ecuación 2

\section{Ecuación 2}

$$
\% \mid B=\left[100 *\left(\frac{(\text { bilatera })}{\text { unilateral izquierdo }+ \text { unilateral derecho }}\right)\right]-100
$$

Se indicó una facilitación bilateral si el IB fue significativamente mayor que 0 , y se indicó un déficit bilateral si el IB fue significativamente menor que 0 . La presencia de un DBL se consideró una diferencia significativa de tareas bilateral es en comparación con la suma de las tareas unilaterales. Este análisis se utilizó recientemente con atletas de judo (Turnes et al., 2019; Kons et al., 2020).

Sprint de 0 a $5 \mathrm{~m}$ (5-M). Para la ejecución de la prueba de velocidad lineal de sprint 5-M, se colocaron dos pares de fotocélulas (Brower Timing System, Salt Lake City, UT) con precisión de 0.001 s a distancias de cero, $5 \mathrm{~m}$ de manera lineal a lo largo del recorrido de sprint. Losatletas corrieron dosveces comenzando desde una posición de pie $0.3 \mathrm{~m}$ detrás de la línea de partida en la prueba tradicional, y $5 \mathrm{~m}$ detrás de la línea de partida en la prueba de inicio. Para evitar las influencias climáticas, las evaluaciones se realizaron en un gimnasio cerrado con piso de madera. Un intervalo de descanso de dos min fue permitido entre los dos intentos, el tiempo más rápido fue considerado para el posterior análisis estadístico.

\section{Procedimientos}

Las evaluaciones fueron realizadas posterior a $24 \mathrm{~h}$ del último entrenamiento físico durante la última semana del mes de abril del 2019. Todas las pruebas 
estandarizadas fueron ejecutadas en un gimnasio techa do con piso de madera y real izadas por el mismo investigador quien estuvo cegado de los atletas del estudio al momento de las evaluaciones. Entre las condiciones de vestimenta se solicitó a los atletas utilizar camiseta deportiva, pantalón corto y calzado para correr. Durante el primer día se evaluó la edad, estatura, y la masa corporal en estado de ayuno. Posteriormente, al díasiguiente, las variables del RF. Previo a las evaluaciones del RF todos los atletas fueron instruidos para que (i) descansa ran de manera adecuada la noche anterior durmiendo 8 h, (ii) no consumieran bebidas estimulantes previa las evaluaciones, (iii) mantuvieran sus hábitos alimentarios y de hidratación habituales. Durante el día de las eva luaciones del RF los atletas previamente fueron instruidos para dar su máximo esfuerzo durante las pruebas. Un calentamiento típico en este deporte fue realizado con $\sim 15$ min de duración que consistió en movilidad articular, trote suave por $\sim 5$ min y estiramientos diná micos. Se realizaron dos ensayos de cada prueba, con una recuperación de $60 \mathrm{~s}$ entre saltos y dos min entre las pruebas CO DS y 5-M (Bishop et al., 2019a).

\section{Análisis estadístico}

Todos los resultados de los atletas analizados son presentados utilizando la media \pm desviación estándar (DE). La normalidad de los datos fue verificada utilizando la prueba de Shapiro-W ilk y la homocedasticidad de varianza a través de la prueba de Levene. Para determinar las diferencias entre el rendimiento del RSBJ con el RSBJ se realizó una pruebaT de Student no pa reado. Posteriormente, para examinar la relación de la $A B$ y el $D B L$ del SBJ con el rendimiento de CODS y 5$M$, además de la relación entre el rendimiento del $S B$ ), RSBJ y RSBJ con el rendimiento del CODS y 5-M se utilizó la prueba de correlación de Pearson ( $r$ ) con el $95 \%$ de coeficiente de intervalo $(95 \% \mathrm{Cl})$. A su vez, el coeficiente de determinación $\left(R^{2}\right)$ fue determinado para expresar el porcentaje de cambio entre las variables analizadas (Borda Pérez et al., 2013). La magnitud de lascorrelaciones fue interpretadautilizando lad deC ohen utilizando los criterios propuestos por Hopkins et al., (2009). De 0 a .30 se consideró [baja]; . 31 a.49 [moderada]; . 50 a . 69 [grande]; .70 a . 89 [muy grande]; y .90 a 1.0, una correlación [casi perfecta a perfecta]. De forma complementaria, para contrastar las hipótesis esta dísticas, se realizó un análisis bayesiano, donde los factores de Bayes $\mathrm{FB}_{10}$ (a favor de la hipótesis alterativa $0 \mathrm{H}_{1}$ ) y $\mathrm{FB}_{0.1}$ (a favor de la hipótesis nula $0 \mathrm{H}_{0}$ ) con el $95 \% \mathrm{Cl}$ fueron calculados (Marsman \& Wagenmakers, 2017;
Q uintana \& W illiams, 2018; Santos-Fernandez, Wu, \& Mengersen, 2019). La interpretación de los resultados fue realizada de acuerdo según la clasificación de los valores de Jeffreys donde para $\mathrm{H}_{1}$ : 1 a 3 se consideró [débil], 3 a 10 [moderado] y > 10 [fuerte] (van Doorn et al. , 2020). Para el análisis estadístico se utilizó el pa quete estadístico GraphPad Prism (versión 6.0, San Diego, California) con excepción de los factores bayesianos los cuales fueron evaluados mediante el pa quete estadístico JASP (Equipo JASP, Versión 0.14, Universidad de Amsterdam). El nivel de significancia esta dística fue establecido en $p<.05$.

\section{Resultados}

Todos los datos anal izados cumplieron con el supuesto de homogeneidad de varianza $(p>98)$ y con con el supuesto de normalidad ( $p>.05$ ).

En relación al rendimiento entre $L S B J$ con $R S B$ J de los atletas no se reportaron diferencias significativas (t $=.47 ; p=.63 ; d=.22$ ) entre ambas extremidades inferiores analizadas.

Latabla 1 describe las características antropométricas $y$ de rendimiento físico de los atletas analizados $(n=$ 10). Los atletas tenían una edad de $14 \pm 1$ años, una estatura de $164 \pm 11 \mathrm{~cm}$ y una masa corporal de $56.5 \pm$ $12.1 \mathrm{~kg}$ En términos de rendimiento físico los atletas obtuvieron un rendimiento en el SBJ de $163.6 \pm 25$ $\mathrm{cm}$, en LSBJ de $127.9 \pm 21 \mathrm{~cm}$, y en RSBJ de $132.8 \pm$ $22.2 \mathrm{~cm}$. Se observó una $A B$ de $5.1 \pm 3.6 \%$ entre ambas extremidades inferiores y un DBL de $-36.9 \pm$ $6.2 \%$. En tanto, obtuvieron un rendimiento en $5-\mathrm{M}$ de $1.26 \pm 0.13 \mathrm{~m} \mathrm{~s}^{1}$ y en la prueba de CODS de $6.96 \pm$ $0.73 \mathrm{~s}$.

La tabla 2 describe en detalle las correlaciones documentadas en este estudio. Entre los principales ha llazgos se reportaron correlaciones significativas inversas [casi perfectas] entre el rendimiento de CODS con el RSB) $\left(r=-.92 ; R^{2}=.85 \% ; p=.00\right)$ y [muy grandes] con SBJ $\left(r=-.87 ; R^{2}=.75 \% ; p=.00\right)$ y con LSBJ $(r=$ $\left.-.82 ; R^{2}=.67 \% ; p=.00\right)$. Una correlación [grande] entre el índice del DBL con $5-M\left(r=.51 ; R^{2}=.26 \%\right.$; $p=.16)$ y [baja] con CODS $\left(r=.20 ; R^{2}=.04 ; p=.60\right)$. Por otra parte, se documentaron correlaciones [moderadas] entre laAB con CODS $\left(r=-.38 ; R^{2}=.15 \% ; p=\right.$ .30) y $5-M\left(r=-.47 ; R^{2}=.22 \% ; p=.19\right)$

En relación a los resultados complementarios según el análisis bayesiano, probabilidades [fuertes] de aceptar la $\mathrm{H}_{1}$ fueron observadas en el $\mathrm{CODS}$ con $\left.\mathrm{RSB}\right)\left(\mathrm{FB}_{10}=\right.$ $151.4 \%)$, probabilidades [moderadas] con SB] $\left(\mathrm{FB}_{10}=\right.$ 
$39.6 \%)$ y LSBJ $\left(\mathrm{FB}_{10}=17 \%\right)$. En tanto probabilidades [bajas] se reportadas con 5-M (FB $\left.{ }_{10}=.19 \%\right)$. Probabilidades [bajas] de aceptar la $\mathrm{H}_{1}$ se observaron entre la $A B$ con el CODS $\left(\mathrm{FB}_{10}=1.0 \%\right)$ y con $5-\mathrm{M}\left(\mathrm{FB}_{10}=1.4 \%\right)$. Final mente, probalidades [moderadas] de aceptar la $\mathrm{H}_{0}$ se documentaron entre el DBL con CODS $\left(\mathrm{FB}_{0.1}=\right.$ $3.4 \%)$ y con $5-\mathrm{M}\left(\mathrm{FB}_{0.1}=5.0 \%\right)$.

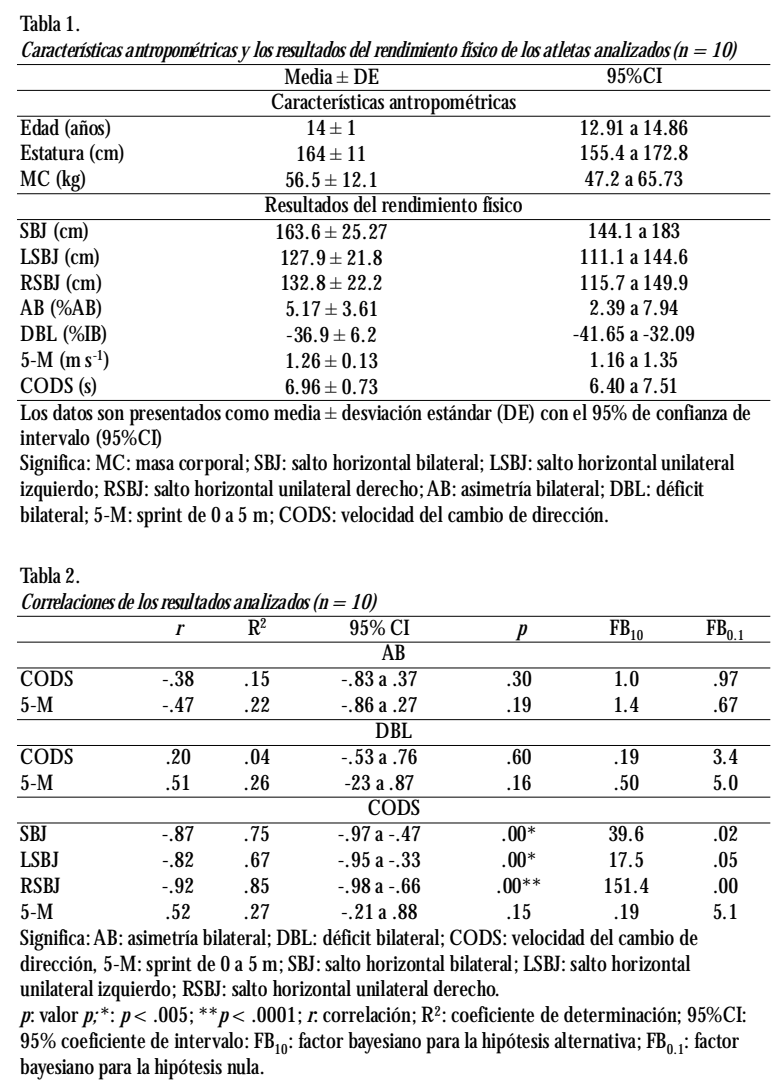

\section{Discusión}

El presente estudio tuvo como propósito principal fue examinar la correlación entre la Asimetría Bilateral y el Déficit Bilateral con la velocidad del cambio de dirección y el sprint en 0 a $5 \mathrm{~m}$, así como entre el salto horizontal con el velocidad del cambio de dirección y el sprint en 0 a $5 \mathrm{~m}$. Entre los principales hallazgos se reportó una baja $A B$ y un $D B L$. Además, se reportaron correlaciones moderadas entre la AB con CODS y 5 $M$. En tanto, correlaciones moderadas entre DBL con 5-M y bajas con CODS. Finalmente, correlaciones significativas entre CODS con los resultados de RSB], SBJ $y L S B]$.

Analizando los resultados principales, se reportó ausencias de correlaciones significativas $(p>.05)$ entre la AB a partir del SBJ con el CODS y 5-M. Estos datos son similares con los reportados por Lockie et al. (2014) en atletas jóvenes de deportes de equipo, quienes no documentaron correlaciones significativas ( $p>.05$ ) entre la $A B$ evaluada mediante SB) y salto vertical, con una disminución sustancial del rendimiento en CODS (PruebaT y 505) y con sprint de $20 \mathrm{~m}$ (con interval os de $0,5 \mathrm{~m}$ y $10 \mathrm{~m}$ ). De manera similar, recientemente los autores Madruga-Parera et al. (2020) en jugadores jóvenes de bal onmano analizaron laAB mediante el SB], CM J, salto lateral, tareas específicas en CODS ( $90^{\circ}$ y $180^{\circ}$ relizadas con dispositivo isonercial). Entre los resultados no documentaron correlaciones significativas $(p>.05)$ relevantes entre la AB mediante SBJ, CMJ y salto lateral con una disminución del rendimiento en las tareas de CO DS 90 y 180 y sprint en $20 \mathrm{~m}$. Aunque, evidenciaron correlaciones significativas $(p<.05)$ con las tareas de CODS con paso lateral y cruzado. Por otro lado, los datos de este estudio difieren con los resulta dos de los autores Bishop et al. (2019b) quienes reportaron $A B$ mediante $C M J$ en futbolistas jóvenes de diferentes categorías de edad, reportando correlaciones inversas significativas $(p<.05)$ con el rendimiento físico en sprint en $5 \mathrm{~m}, 10 \mathrm{~m}, 20 \mathrm{~m}$ y CODS (através de la prueba 505) ejecutado inicial mente con la pierna derecha y posteriormente con la pierna izquierda en futbolistas de diferentes categorías de edad. En deportes de combate, analizando laAB con tareas específicas de rendimiento, principalmente en judo, se han reportado diferencias significativas $(p<.05)$ en indicadores de la prueba Special Judo FitnessTest con el lado dominante vsno-dominante (Konset al., 2020) y un incremento en la magnitud de la $A B$ mediante el SBJ tras cuatro combates sucesivos (Kons et al., 2021).

De acuerdo con lo anterior, la ausencia de correlaciones significativas en los resultados de este estudio podrían ser explicados por la bajaAB (5.1\%; $p=.63$; $d$ $=.22$ ) reportada entre ambas extremidades inferiores de los atletas analizados. Además, se sugiere que la $A B$ estaría influenciada por la edad y por el tipo de prueba real izada (Sarabon et al., 2020; M adruga-Parera et al., 2020; Bishop et al., 2017).

En cuanto al DBL se documentaron ausencias de correlaciones significativas $(p>.05)$ con el rendimiento en CODS y $5-M$. Estos resultados contrastan parcialmente con los documentados por Bishop et al. (2019a) quienes en estudiantes activos universitarios reportaron presencia de DBL a patir del SBJ, CMJ y DJ y correlaciones inversas significativas $(p<.05)$ con un ma yor rendimiento en CODS (prueba 505) de manera bilateral y ejecutada iniciando con la pierna izquierda y posteriormente con la pierna derecha. A su vez, los autores, no documentaron correlaciones significativas ( $p$ 
$>$.05) con sprint en $10 \mathrm{~m}$ y $30 \mathrm{~m}$. Losautores, sugieren que la presencia del DBL de los participantes analizados sería beneficioso para el rendimiento de CODS por la naturaleza unilateral de esta habilidad. En este sentido, las correlaciones inversas significativas $(p=.00)$ entre el SBJ y mayores con la extremidad inferior derechavs. la extremidad inferior izquierda con el rendimiento CODS documentadas en este estudio, además, una probabilidad baja de aceptar la $\mathrm{H}_{0}\left(\mathrm{FB}_{0.1}=3.0\right)$ podrían apoyar este planteamiento. Sin embargo, se necesitan más estudios para ser concluyentes.

También, correlaciones significativas $(p<.05)$ entre el SBJ con el rendimiento en CODS fueron reportados en este estudio. Esto es consistente con la reciente evidencia en karate (HerreraValenzuela et al., 2020) y taekwondo (0 jeda-Aravena et al., 2020; Chaabene et al., 2018) donde se documentó una correlación entre pruebas de CODS específicas de sus respectivos deportes con pruebas indirectas de fuerza explosiva, incluyendo SBJ. En este sentido, los autores señalan que la fuerza explosiva es un factor relevante para el rendimiento en los deportes de combate y debería entrenarse tanto el componente de fuerza de reacción al suelo horizontal como vertical de los saltos ya que ambos podrían mejorar el rendimiento del CODS (Chaabene et al., 2018). A su vez, se reportó un predominio unila teral de la extremidad derecha manifestado en el rendimiento obtenido en RSBJ con una correlación inversa con el rendimiento en CODS $(p<.00)$. En este sentido, las pruebas de CO DS presentan una aceleración en el plano horizontal que podría tener relación con el rendimiento de salto horizontal unilateral (Lockie et al., 2014). En el karate en particular, las respuestas de este estudio serían esperables, dado el predominio unilateral de la guardia izquierda con utilización del segmento derecho documentado en combates oficiales (Ibáñez et al., 2018) siendo indicativo de una preferencia por la predominancia unilateral del deporte.

Por otra parte, este estudio no estuvo exento de limitaciones. Es importante señalar que los análisis de correlación tienen limitaciones, ya que factores como la edad de los atletas analizados, la masa corporal, los atributos de la condición física, la flexibilidad, la técnica y la fuerza muscular de las piernas pueden tener un efecto en los modelos estadísticos derivados (Lockie et al., 2014) y por tanto los resultados deben ser interpretados con precaución. También hay que considerar que el bajo número de atletas producto de la homogeneidad de la muestra, no permite extrapolar los hallazgos, requiriendo de futuros estudios con mayor poten- cia estadística para ser concluyentes.

Futuros estudios podrían comparar laAB y el DBL utilizando diferentes tipos de saltos, y diferente ecuaciones descritas en la literatura (Bishop et al. 2019a; Bishop et al. , 2018b), utilizando diseños de mayor complejidad (por ej. test-retest) y medidas de confiabilidad (correlación intra-clase, coeficiente de variación y error técnico de medición). Lo anterior, con el fin de estudiar en profundidad el impacto de laAsimetría Bilateral y el Déficit Bilateral y su relación con el rendimiento físico en los deportes de combate.

\section{Aplicaciones prácticas}

En términos prácticos la evaluación de las pruebas del salto bilateral y unilateral horizontal pueden ser utilizados por los entrenadores de atletas de karate para (i) monitorear la fuerza explosiva con vector horizontal (ii) determinar laAB y la IB con la finalidad de prevenir una exhacerbada asimetría y con ello el riesgo de lesión (iii) utilizar el salto largo horizontal bilateral y unilateral como un método de fácil aplicación y de bajo costo de implementación y facilidad de medición.

\section{Conclusiones}

La Asimetría Bilateral y el Déficit Bilateral se rela cionarían negativamente con el rendimiento de la velocidad del cambio de dirección y el sprint en $5 \mathrm{~m}$ en los atletas de karate de categoría cadetes. Aunque, el rendimiento de la velocidad del cambio de dirección se relaciona con el rendimiento de la fuerza explosiva horizontal bilateral y unilateral.

\section{Referencias}

Bishop, C., Berney, J., Lake, J., Loturco, I., Blagrove, R., Turner, A., \& Read, P. (2019a). Bilateral Deficit During JumpingTasks: RelationshipW ith Speed and Change of Direction Speed Performance. The Journal of Strength \& Conditioning Research, Publish Ahead of Print. https:/ / doi.org/ 10.1519/ JSC.0000000000003075

Bishop, C., Brashill, C., Abbott, W., Read, P., Lake, J., \& Turner, A. (2019b). Jumping Asymmetries Are AssociatedW ith Speed, Change of Direction Speed, and Jump Performance in Elite Academy Soccer Players. The Journal of Strength \& Conditioning Research, Publish Ahead of Print. https: / / doi.org/ 10.1519/ JSC.0000000000003058

Bishop, C., Lake, J., Loturco, I., Papadopoulos, K., Turner, 
A., \& Read, P. (2018a). Interlimb Asymmetries: The Need for an Individual Approach to Data Analysis. The Journal of Strength \& Conditioning Research, 35(3), 695701. https/ / doi.org/ 10.1519/ JSC.0000000000002729 Bishop, C., Read, P., Chavda, S. Jarvis, P., \& Turner, A. (2019c). Using unilateral strength, power and reactive strength tests to detect the magnitude and direction of asymmetry: A test-retest design. Sports, 7(3), 58. https:/ / doi.org/ 10.3390/ sports7030058

Bishop, C., Read, P., Lake, J., Chavda, S., \& Turner, A. (2018b). Interlimb asymmetries: Understanding how to calculate differencesfrom bilateral and unilateral tests. Strength \& Conditioning Journal, 40(4), 1-6. https:// doi.org/ 10.1519/ SSC.0000000000000371

Bishop, C., Turner, A., \& Read, P. (2017). Effects of interlimb asymmetries on physical and sports performance: A systematic review. Journal of Sports Sciences, 36(10), 1135-1144. https:/ / doi.org/ 10.1080/ 02640414.2017 .1361894

Bishop, C., Turner, A., Maloney, S., Lake, J., Loturco, I., Bromley, T., \& Read, P. (2019d). Drop jump asymmetry is associated with reduced sprint and change-of-direction speed performancein adult female soccer players. Sports, 7(1), 29. https: / / doi.org/ 10.3390/ sports7010029

BordaPérez, M., TuescaM olina, R., \& N avarro Lechuga, E. (2013). M étodoscuantitativos. Herramientas para la investigación en salud 4 ed. Universidad del Norte.

Caballero, P. G. \& \& Díaz, J. C. (2003). M anual deAntropometría. Cuba.

Chaabène, H., Franchini, E., Sterkowicz, S., Tabben, M., Hachana, Y., \& Chamari, K. (2015). Physiological responses to karate specific activities. Science $\&$ Sports, 30(4), 179-187. https:// doi.org/ 10.1016/ j.scispo.2015.03.002

Chaabene, H., Hachana, Y., Franchini, E., Mkaouer, B., \& Chamari, K. (2012). Physica and physiological profile of elite karate athletes. Sports mediane, 42(10), 829-843. https:/ / doi.org/ 10.1007/ BF03262297

Chaabene, H., Negra, Y., Capranica, L., Bouguezzi, R., Hachana,Y., Rouahi, M.A., \& M kaouer, B. (2018).Validity and reliability of a new test of planned agility in elite taekwondo athletes. The Journal of Strength \& Conditioning Research, 32(9), 2542-2547. https:/ / doi.org/ 10.1519/ JSC. 0000000000002325

de Q uel, Ó. M., Ara, I., Izquierdo, M., \& Ayán, C. (2020). Does Physical Fitness Predict Future Karate Success? A Study inYoung Female Karatekas. International Journal of SportsPhysiology and Performance, 15(6), 868-873. https:/ / doi.org/ 10.1123/ ijspp.2019-0435

Fandos Soñén, D., Facón Miguel, D., M oreno Azze, A., \&
Pradas de LaFuente, F. (2020). Influenciade un entrenamiento pliométrico monopoda y bipodal sobre la fuerzaexplosivadel tren inferior y lacorrección de asimetrías en karatekas. Retos, 39, 367-371. https:/ / doi. org/ 10.47197/ retos. v0i39. 78818

Herrera-Valenzuela, T., Miccono-González, G., FazekasMolina, M., AstorgarRojas, G., Valdés-Badilla, P., Ojeda Aravena, A., \& Franchini, E. (2020). Relación entre el Movement change in karate position Test con el rendimiento neuromuscular en atletas de karate: Un estudio piloto. Retos, 39, 505-508. https:/ / doi.org/ 10.47197/ retos. v0i39.81409

Hoffman, J. R., Ratamess, N. A., Klatt, M., Faigenbaum, A. D., \& Kang, J. (2007). Do bilateral power deficits influence direction-specific movement patterns? Research in Sports Medicine, 15(2), 125-132. https:/ / doi. org/ 10.1080/15438620701405313

Hopkins,W. G., Marshall, S.W. , Batterham, A. M., \& Hanin, J. (2009). Progressive statistics for studies in sports me dicine and exercise science. Mediane and Science in Sports and Exercise, 41(1), 3-13. https:/ / doi.org/ 10.1249/ MSS.0b013e31818cb278

Ibáñez, R. , Lapresa, D. ,Arana, . . Camerino, 0., \&Anguera, M. (2018).Análisisobservacional del combatede karae: desempeño técnico-táctico del competidor de élite. Cultura, Ciencia y Deporte, 13(37), 61-70. https:/ / dx.doi.org/ 10.12800/ ccd.v13i37.1039

Impellizzeri, F. M., Rampinini, E., Maffiuletti, N., \& Marcora, S. M. (2007). A vertical jump force test for assessing bilateral strength asymmetry in athletes. $\mathrm{Me}$ dicineand Sciencein Sportsand Exercise, 39 (11), 2044-2050. https:/ / doi.org/ 10.1249/ mss.0b013e31814fb55c

Kons, R. L., Diefenthæeler, F., O rssatto, L. B. R., Sakugawa, R. L., da Silva Junior, J. N., \& Detanico, D. (2020). Relationship between lower limb asymmetry and judospecific test performance. Sport Sciences for H ealth, 16(2), 305-312. https:/ / doi.org/ 10.1007/ s11332-019-006065

Kons, R. L., Pupo, J. D., Gheller, R. G., Costa, F. E., Rodrigues, M. M., Bishop, C., \& Detanico, D. (2021). Effects of successive judo matches on interlimb asymmetry and bilateral deficit. Physical Therapy in Sport, 47,15-22. https:/ / doi.org/ 10.1016/ j.ptsp. 2020.10.007 Little,T., \&W illiams, A. G. (2005). Specificity of acceleration, maximum speed, and agility in professional soccer players. Journal of Strength and Conditioning Rescarch, 19(1), 76-78. https./ / doi.org/ 10.1519/ 14253.1

Lockie, R. G., Callaghan, S. J., Berry, S. P., Cooke, E. R., Jordan, C. A., Luczo, T. M., \& Jeffriess, M. D. (2014). Relationship between unilateral jumping ability and 
asymmetry on multidirectional speed in team-sport athletes The Journal of Strength \& Conditioning Research, 28(12), 3557-3566. https:/ / doi. org/ 10.1519/ JSC. 0000000000000588

Loturco, I., Nimphius, S., Kobal, R., Bottino, A., Zanetti, V., Pereira, L. A., \& Jeffreys, I. (2018). Change-of direction deficit in elite young soccer players. German Journal of Exercise and Sport Ressearch, 48(2), 228-234. https:/ / doi.org/ 10.1007/ s12662-018-0502-7

Madruga-Parera, M., Bishop, C., Beato, M., FortVanmeerhæeghe, A., Gonzalo-Skok, 0., \& RomeroRodríguez, D. (2021). Relationship Between Interlimb Asymmetries and Speed and Change of Direction Speed in Youth Handball Players. The Journal of Strength \& Conditioning Research, Publish Ahead of Print. https:/ / doi.org/ 10.1519/ JSC.0000000000003328

Marsman, M., \& Wagenmakers, E.--. (2017). Bayesian benefits with JASP. European Journal of Developmental Psychology, 14(5), 545-555. https:/ / doi.org/ 10.1080/ 17405629.2016.1259614

McLester, C. N., Nickerson, B. S., Kliszczewicz, B. M., \& McLester, J. R. (2020). Reliability and Agreement of Various InBody Body Composition Analyzers as Compared to Dual-Energy X-Ray Absorptiometry in Healthy Men andWomen. Journal of Clinical Densitometry, 23(3), 443-450. https:/ / doi.org/ 10.1016/ j.jocd. 2018.10.008

Meyers, R. W., Oliver, J. L., Hughes, M. G., Lloyd, R. S., $\&$ Cronin, J. B. (2017). Asymmetry during maximal sprint performance in 11-to 16-year-old boys. Pediatric exercisescience, 29(1), 94-102. https:/ / doi.org/ 10.1519/ JSC.0000000000002608

O jeda-Aravena, A. , Azócar-Gallardo, J., HérnandezMosqueira, C., \& HerreraValenzuela, T. (2020). Rela ción entre la pruebade agilidad específica en tæekwondo (tsat), la fuerza explosiva y la velocidad líneal en 5-m en atletas de trekwondo de ambos sexos. Retos 39, 84-89. https:/ / doi.org/ 10.47197/ retos. V0i39. 78395

Quintana, D. S., \& W illiams, D. R. (2018). Bayesian aternativesfor common null-hypothesissignificancetests in psychiatry: A non-technical guide using JASP. BMC Psychiatry, 18(1), 178. https/ / doi.org/ 10.1186/ s12888018-1761-4

Sannicandro, I., Rosa, R. A., De Pascalis, S., \& Piccinno, A. (2012). The determination of functional asymmetries in the lower limbs of young soccer players using the countermovement jump. The lower limbs asymmetry of young soccer players. Science \& sports, 27(6), 375- 377. https:/ / doi.org/ 10.1016/ j.scispo.2011.11.001

Santos-Fernandez, E., W u, P. \& Mengersen, K. (2019).
Bayesian statistics meets sports: a comprehensive review. Journal of Quantitative Analysis in Sports, 15(4), 289-312. https:/ / doi.org/ 10.1515/ jqas-2018-0106

Sarabon, N., Kozinc, Z., Bishop, C., \& Maffiuletti, N. A. (2020). Factors influencing bilateral deficit and interlimb asymmetry of maximal and explosive strength: Motor task, outcome measure and muscle group. European Journal of Applied Physiology, 120 (7), 1681-1688. https/ / doi.org/ 10.1007/ s00421-020-04399-1

Scattone-Silva, R. , Lessi, G. C., Lobato, D. F. M., \& Serrão, F. V. (2012). A cceleration time, peak torque and time to peak torquein elitekarateathletes. Science \& Sports, 27(4), e31-e37. https:/ / doi. org/ 10.1016/ j.scispo. 2011.08.005

Tabben, M., Miarka, B., Chamari, K., \& Beneke, R. (2018). Decisive moment: A metric to determine success in elite karate bouts. International journal of sports physiology and performance, 13(8), 1000-1004. https:/ / doi.org/ 10.1123/ ijspp. 2017-0526.

Turnes, T., Silva, B. A., Kons, R. L., \& Detanico, D. (2021). IsBilatera Deficit in Handgrip StrengthA ssociatedW ith Performance in Specific JudoTasks? The Journal of Strength \& Conditioning Research, Publish Ahead of Print. https:/ / doi.org/ 10.1519/ JSC.0000000000003441

van Doorn, J., van den Bergh, D., Böhm, U., Dablander, F., Derks, K., Draws, T., Etz, A., Evans, N. J., Gronau, Q. F., Haaf,J. M., Hinne, M., Kucharský, Š, Ly, A., Marsman, M., Matzke, D., Gupta, A. R. K. N., Sarafoglou, A., Stefan, A., Voelkel, J. G., \&Wagenmakers, E.-J. (2020). The JASP guidelines for conducting and reporting a Bayesian analysis. Psychonomic Bulletin $\&$ Review. https: / / doi.org/ 10.3758/ s13423-020-01798-5 van Doorn, J., van den Bergh, D., Böhm, U., Dablander, F., Derks, K., Draws, T., ... Haaf, J. M. (2020). The JASP guidelines for conducting and reporting a Bayesian analysis. Psychonomic Bulletin \& Review, 1-14.

World M edicalAssociation. (2013).WorldM edicalAssociation Declaration of Helsinki: Ethical principles for medical researchinvolving human subjects. JAM A, 310(20), 21912194. https:/ / doi.org/ 10.1001/ jama.2013.281053

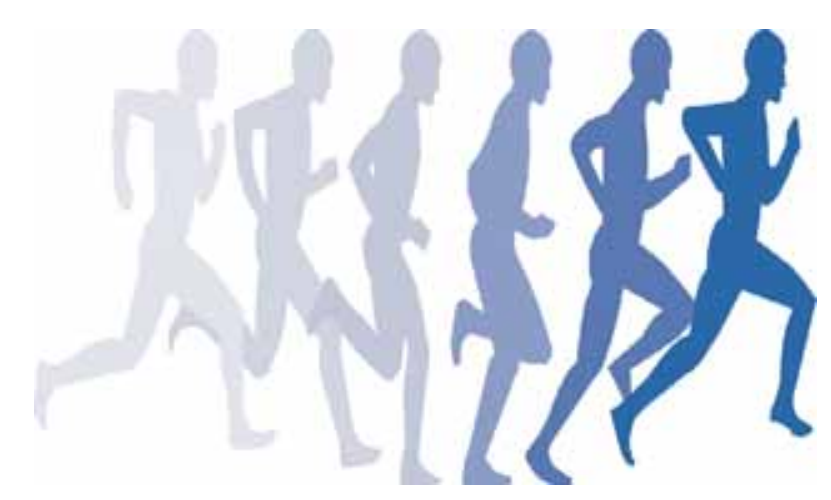

